\title{
Forward-backward asymmetry in the gauge-Higgs unification at the International Linear Collider
}

\author{
Shuichiro Funatsu* \\ Institute of Particle Physics and Key Laboratory of Quark and Lepton Physics (MOE), \\ Central China Normal University, Wuhan, Hubei 430079, China \\ E-mail: funatsu@mail.ccnu.edu.cn
}

Effects of $Z^{\prime}$ in the $S O(5) \times U(1)$ GHU A-model on the fermion pair production processes $e^{+} e^{-} \rightarrow \bar{f} f$ are explained. In the $S O(5) \times U(1)$ GHU model, the Kaluza-Klein excitation modes of $\gamma, Z$ and the neutral $S U(2)_{R}$ gauge boson appear as $Z^{\prime}$ s. There are large asymmetries between $Z^{\prime}$ couplings with left- and right-handed fermions. Although $Z^{\prime}$ mass is $6-8 \mathrm{TeV}$, the significant deviations of the cross sections and forward-backward asymmetries from those in the standard model are predicted at $\sqrt{s}=250 \mathrm{GeV}$ by using the polarised beams.

Corfu Summer Institute 2019 "School and Workshops on Elementary Particle Physics and Gravity" (CORFU2019)

31 August - 25 September 2019

Corfù, Greece

${ }^{*}$ Speaker. 


\section{Introduction}

By the discovery of the Higgs boson at the Large Hadron Collider (LHC), all of the fields in the standard model (SM) were discovered. However, the SM is not a perfect theory. In the SM, the Higgs boson mass is not protected by a symmetry, so there is the fine-tuning problem of the Higgs boson mass. In addition, the reason of the negative Higgs boson mass in the Lagrangian is not explained. Hence studies for the electro-weak sector including the Higgs sector of the SM and of the models beyond the SM are increasingly important.

The gauge-Higgs unification (GHU) is a solution to the fine-tuning problem of the Higgs boson mass [1,2]. In the GHU models, the Higgs boson appears as an extra-dimensional component of the higher-dimensional gauge boson. Hence the Higgs sector is governed by the gauge principle and the Higgs boson mass is protected against the radiative corrections. The Higgs boson is massless at the tree-level and acquires the finite mass by the radiative corrections [3]. In the GHU models, the most important parameter is the Wilson line phase along the compactified extra dimension, $\theta_{H}$. The effective potential is written by the function of $\theta_{H}$. This phase cannot be integrated out because the extra dimension is compactified. This mechanism of dynamical symmetry breaking is called the Hosotani mechanism.

The phenomenologically most viable GHU model is the $S O(5) \times U(1)$ GHU model [4-16]. In the $S O(5) \times U(1)$ GHU model, the Higgs doublet is embedded in fifth dimensional components of the $S O(5) / S O(4)$ gauge boson. Thus the Higgs boson is protected by the custodial symmetry. The most severe constraint on the model comes from the $Z^{\prime}$ searches at the LHC. In this model, the Kaluza-Klein (KK) excitations of $\gamma, Z$ and $Z_{R}$ which is the neutral $S U(2)_{R}$ gauge boson also mediate $e^{+} e^{-} \rightarrow f \bar{f}$ processes. As shown in Ref $[12,13]$, there are large asymmetries between the $Z$ 's couplings with the left- and right-handed quarks and leptons, which are naturally caused from the metric. Because of the large couplings, $Z$ 's have large decay widths and the lower limit of their masses are severely constrained. The parameter region is constrained as $\theta_{H} \lesssim 0.1$, where $m_{\mathrm{KK}} \gtrsim 8 \mathrm{TeV}[11]$.

The International Linear Collider (ILC) has the capabilities for precise measurements of the electroweak sector [17-20] and effects of new physics on the cross sections and the forwardbackward asymmetries of $e^{+} e^{-} \rightarrow f \bar{f}$ processes might be observed [21-24]. The effects of $Z^{\prime}$ bosons in our $S O(5) \times U(1)$ GHU model on these values are studied in Ref. [12]. Because of the large asymmetries of the $Z^{\prime}$ couplings, clear deviations would be seen with the polarised electron beams although $Z^{\prime}$ masses are much higher than the collision energy of the ILC. In the study, not all of the allowed region of parameters is explored. Therefore the effects of $Z^{\prime}$ bosons are more broadly studied in Ref. [13].

In this proceeding, the cross sections and forward-backward asymmetries of the fermion pair production processes mediated by the $Z^{\prime}$ bosons are explained. In Section 2 , the $S O(5) \times U(1)$ GHU models are introduced. In Section 3, properties of the $Z^{\prime}$ bosons in this model is explained. In section 4, the results obtained in Ref. $[12,13]$ are shortly reviewed. In Sect. 5, the results are summarised. 


\section{Model}

The $S O(5) \times U(1)$ GHU models considered in this proceeding are shortly reviewed here. It is defined on the Randall-Sundrum (RS) warped metric [25], its metric is

$$
d s^{2}=g_{M N} d x^{M} d x^{N}=e^{-2 \sigma(y)} \eta_{\mu v} d x^{\mu} d x^{v}+d y^{2},
$$

$(0 \leq|y| \leq+L)$ where $\eta_{\mu v}=\operatorname{diag}(-1,1,1,1) \cdot y=0$ is called the UV brane and $y=L$ is called the IR brane. The region between the two branes are called the bulk space. The metric has a negative curvature, therefore the RS spacetime is an AdS spacetime.

The action has the $S O(5) \times U(1)_{X} \times S U(3)_{C}$ gauge symmetry. The $S O(5), U(1)_{X}$ and $S U(3)_{C}$ gauge fields are expressed as $A_{M}, B_{M}$ and $G_{M}$, respectively. The $S O(5)$ gauge fields $A_{M}$ are decomposed as

$$
A_{M}=\sum_{a_{L}=1}^{3} A_{M}^{a_{L}} T^{a_{L}}+\sum_{a_{R}=1}^{3} A_{M}^{a_{R}} T^{a_{R}}+\sum_{\hat{a}=1}^{4} A_{M}^{\hat{a}} T^{\hat{a}},
$$

where $T^{a_{L}, a_{R}}\left(a_{L}, a_{R}=1,2,3\right)$ and $T^{\hat{a}}(\hat{a}=1,2,3,4)$ are the generators of $S O(4) \simeq S U(2)_{L} \times S U(2)_{R}$ and $S O(5) / S O(4)$, respectively. There are two types of the fermion multiplets in this model. $\Psi_{a}^{\alpha}(a=1,2,3,4$ and $\alpha$ represents the generations) are the four $S O(5)$-vector (5 representation) fermions and $\Psi_{F}^{\delta}\left(\delta=1, \cdots, N_{F}\right)$ are $N_{F}$ number of $S O(5)$-spinor (4 representation) fermions, which exist in the bulk space. The colour indices are not shown.

The boundary conditions at $y_{0}=0$ and $y_{1}=L$ are given by

$$
\begin{aligned}
&\left(\begin{array}{c}
A_{\mu} \\
A_{y}
\end{array}\right)\left(x, y_{j}-y\right)=P_{\mathrm{ve}}\left(\begin{array}{c}
A_{\mu} \\
-A_{y}
\end{array}\right)\left(x, y_{j}+y\right) P_{\mathrm{ve}}^{-1}, \\
&\left(\begin{array}{c}
B_{\mu} \\
B_{y}
\end{array}\right)\left(x, y_{j}-y\right)=\left(\begin{array}{c}
B_{\mu} \\
-B_{y}
\end{array}\right)\left(x, y_{j}+y\right), \\
&\left(\begin{array}{c}
G_{\mu} \\
G_{y}
\end{array}\right)\left(x, y_{j}-y\right)=\left(\begin{array}{c}
G_{\mu} \\
-G_{y}
\end{array}\right)\left(x, y_{j}+y\right), \\
& \Psi_{a}^{\alpha}\left(x, y_{j}-y\right)=P_{\mathrm{ve}} \Gamma^{5} \Psi_{a}^{\alpha}\left(x, y_{j}+y\right), \\
& \Psi_{F}^{\delta}\left(x, y_{j}-y\right)=(-1)^{j} P_{\mathrm{sp}} \Gamma^{5} \Psi_{F}^{\delta}\left(x, y_{j}+y\right), \\
& P_{\mathrm{ve}}=\operatorname{diag}(-1,-1,-1,-1,+1), \\
& P_{\mathrm{sp}}=\operatorname{diag}(+1,+1,-1,-1) .
\end{aligned}
$$

By these boundary conditions, $A_{\mu}^{a_{L}}, A_{\mu}^{a_{R}}$ and $A_{y}^{\hat{a}}$ have the zero modes. Thus, the $S O(5) \times U(1)_{X}$ symmetry is broken to $S O(4) \times U(1)_{X} \simeq S U(2)_{L} \times S U(2)_{R} \times U(1)_{X}$ at low energy and the Higgs boson is protected by the custodial symmetry. The remaining $S U(2)_{R}$ symmetry must be broken because no $S U(2)_{R}$ gauge bosons have been observed. In our model the $S U(2)_{R} \times U(1)_{X}$ symmetry is broken to $U(1)_{Y}$ by the brane interaction with a brane scalar on the UV brane. Finally the remaining $S U(2)_{L} \times U(1)_{Y}$ is broken to $U(1)_{\mathrm{EM}}$ by the Hosotani mechanism. For quarks and leptons, the bidoublets have the left-handed zero modes and the singlets have the right-handed zero modes. 
The vacuum expectation value of the Higgs boson appears as a Wilson line phase. The vacuum expectation value is taken to $\left\langle A_{y}^{\hat{a}}\right\rangle=v \cdot \delta^{a 4} \tilde{u}_{H}(y)$, where $\tilde{u}_{H}(y) \equiv \sqrt{\frac{2 k}{e^{2 k L}-1}} e^{2 k y}$, and the Wilson line phase is given by

$$
\exp \left\{\frac{i}{2} \theta_{H} 2 \sqrt{2} T^{\hat{4}}\right\}=\exp \left\{i g_{A} \int_{0}^{L} d y\left\langle A_{y}\right\rangle\right\} .
$$

$v$ is written as $v=f_{H} \theta_{H}$, where

$$
f_{H} \equiv \frac{2}{g_{A}} \sqrt{\frac{k}{e^{2 k L}-1}} .
$$

It is thought that there is a holography between the $S O(5) \times U(1)$ GHU models and the minimal composite Higgs models [4,5]. A composite pseudo Nambu-Goldstone boson in composite Higgs models corresponds to a zero-mode of $A_{y}$ in GHU models defined on AdS spacetime and the Higgs potential in composite Higgs models correspond to the effective potential by the Hosotani mechanism in GHU models. Here it should be emphasised that the effective potential in GHU models are calculable. In addition, actions in GHU models are more restrictive.

Recently, two types of the $S O(5) \times U(1)$ GHU models are phenomenologically studied. One is called the A-model and the other is called the B-model. The main difference is the $S O(5)$ representations in which the quarks and leptons are embedded. In this proceeding, the A-model is explained.

Table 1: Matter and brane fields of the $S O(5) \times U(1)$ GHU A-model and B-model. The left numbers $(\mathbf{3}$ or 1) in the parenthesis represent the representation for $S U(3)_{C}$ (colour triplets or singlets). The right numbers represent the representations for $S O(5)$. For the brane fields, $[2, \mathbf{1}]$ is $S U(2)_{L}$ doublets and $S U(2)_{R}$ singlets and $[1,2]$ is $S U(2)_{L}$ singlets and $S U(2)_{R}$ doublets. The subscripts represent the $U(1)_{X}$ charges.

\begin{tabular}{|c||c|c|}
\hline & A model & B model \\
\hline \hline quark & $\Psi_{1}^{\alpha}:(\mathbf{3}, \mathbf{5})_{+\frac{2}{3}}, \Psi_{2}^{\alpha}:(\mathbf{3}, \mathbf{5})_{-\frac{1}{3}}$ & $\Psi_{(\mathbf{3}, \mathbf{4})}^{\alpha}:(\mathbf{3}, \mathbf{4})_{+\frac{1}{6}}, \Psi_{(\mathbf{3}, \mathbf{1})}^{ \pm \alpha}:(\mathbf{3}, \mathbf{1})_{-\frac{1}{3}}^{ \pm}$ \\
\hline lepton & $\Psi_{3}^{\alpha}:(\mathbf{1}, \mathbf{5})_{-1}, \Psi_{4}^{\alpha}:(\mathbf{1}, \mathbf{5})_{0}$ & $\Psi_{(\mathbf{1}, \mathbf{4})}^{\alpha}:(\mathbf{1}, \mathbf{4})_{-\frac{1}{2}}$ \\
\hline dark fermion & $\Psi_{F}^{\delta}:(\mathbf{1}, \mathbf{4})_{+\frac{1}{2}}$ & $\Psi_{F}^{\beta}:(\mathbf{3}, \mathbf{4})_{+\frac{1}{6}}, \Psi_{(\mathbf{1}, \mathbf{5})}^{ \pm \gamma}:(\mathbf{1}, \mathbf{5})_{0}^{ \pm}$ \\
\hline \hline brane fermion & $\hat{\chi}_{1,2,3 R}^{q}:(\mathbf{3},[\mathbf{2}, \mathbf{1}])_{+\frac{7}{6},+\frac{1}{6},-\frac{5}{6}}$ & $\chi^{\alpha}:(\mathbf{1}, \mathbf{1})_{0}$ \\
\hline brane scalar & $\hat{\chi}_{1,2,3 R}^{l}:(\mathbf{1},[\mathbf{2}, \mathbf{1}])_{-\frac{3}{2},+\frac{1}{2},-\frac{1}{2}}$ & \\
\hline symmetry of & $\hat{\Phi}:(\mathbf{1},[\mathbf{1}, \mathbf{2}])_{+\frac{1}{2}}$ & $\Phi_{(\mathbf{1}, \mathbf{4})}:(\mathbf{1}, \mathbf{4})_{+\frac{1}{2}}$ \\
\hline $\begin{array}{c}\text { brane interactions } \\
\text { bra }\end{array}$ & $S U(3)_{C} \times S O(4) \times U(1)_{X}$ & $S U(3)_{C} \times S O(5) \times U(1)_{X}$ \\
\hline
\end{tabular}




\section{Parameters, $Z^{\prime}$ couplings and decay widths}

In the A-model, there are only two free parameters. From the metric, two parameters $k$ and $e^{k L}$ appear. By adjusting $k$, the $Z$-boson mass is determined. The three gauge couplings in the gauge Lagrangian are set to realise 4D couplings. In the quark Lagrangian, two mass parameters appear for each generations. One is the bulk mass $c$ and the other is a ratio of brane masses $r$. The brane interactions are necessary to obtain mass ratio of the up-type and down-type quarks. By adjusting $c$ and $r$, the up- and down-type quark masses are realised for each generations. The dark fermions $\Psi_{F}^{\delta}\left(\delta=1, \cdots, N_{F}\right)$ have the bulk mass $c_{F}$. The dark fermions do not contain the ordinary quarks and leptons, so this $c_{F}$ is set to realise $m_{H}=125 \mathrm{GeV}$. It is already known that Kaluza-Klein scale $m_{K K}$ is almost independent of $N_{F}$ by setting $N_{F}$ and $c_{F}$ to obtain a set of $m_{H}$ and $\theta_{H}$. Therefore the phenomenology of the quark and lepton at low energy is almost uniquely determined by one parameter, $\theta_{H}$.

Table 2: Relevant parameters of the A-model.

\begin{tabular}{c|ccccc}
\hline$\theta_{H}$ & $e^{k L}$ & $\left|c_{t}\right|$ & $\left|c_{F}\right|$ & $k(\mathrm{GeV})$ & $m_{\mathrm{KK}}(\mathrm{GeV})$ \\
\hline 0.10 & $2.90 \times 10^{4}$ & 0.16116 & 0.29617 & $7.4431 \times 10^{7}$ & 8063 \\
0.09 & $1.70 \times 10^{4}$ & 0.11646 & 0.27670 & $4.7190 \times 10^{7}$ & 8721 \\
0.08 & $1.01 \times 10^{4}$ & 0.008914 & 0.25356 & $3.0679 \times 10^{7}$ & 9544 \\
\hline
\end{tabular}

The relevant model parameters of A-model are shown in Table 2. The smaller $\theta_{H}$ corresponds to the higher $m_{K K}$. The upper bound of $\theta_{H}$ (or lower bound of $m_{K K}$ ) is constrained from dilepton searches at the LHC [11]. The lower bound of $\theta_{H}$ is determined by the condition to realise the topquark mass. Because the fermion mass is maximum for $|c|=0$, top-quark mass is not realised for $\theta_{H}<0.08$. The KK scale is defined by $m_{K K} \equiv \pi k /\left(e^{k L}-1\right)$ and $8-10 \mathrm{TeV}$ in the $0.08 \leq \theta_{H} \leq 0.10$ region.

To obtain the low energy effective theory, each fields are expanded as the KK tower. The profile of the fermion mode functions are determined by the bulk mass parameter $c$. Fermion on the warped metric is expanded as [26]

$$
\Psi_{L, R}(x, y)=\frac{e^{\frac{3}{2} k y}}{\sqrt{L}} \sum_{n=0}^{\infty} \psi_{L, R}^{(n)}(x) \frac{f_{L, R}^{(n)}(y)}{\sqrt{N^{(n)}}},
$$

where $N^{(n)}$ is the normalisation factor defined as

$$
N^{(n)}=\int_{0}^{L} \frac{d y}{L}\left\{f_{L, R}^{(n)}(y)\right\}^{2} .
$$

Considering only the zero mode, $f_{L, R}^{(0)}(y)$ is

$$
f_{L, R}^{(0)}(y)=e^{\left(\frac{1}{2} \mp c\right) k y} .
$$

The sign $\mp$ corresponds to the left- and right-handed fermions, respectively. Therefore the lefthanded zero-mode is localised towards $y=0$ and $y=L$ for $\frac{1}{2}<c$ and $c<\frac{1}{2}$. In contrast, the behaviour of the right-handed zero-mode is obtained by changing the sign of $c$ as derived from (3.3). Their behaviour are plotted in Figure 1. 


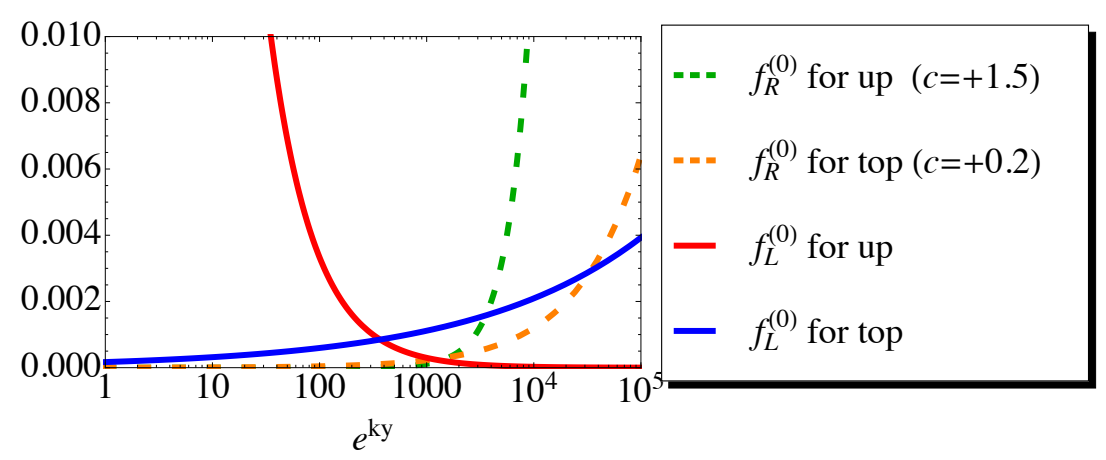

Figure 1: The profile of the fermion mode functions are shown for $0<c$. As shown in Table 2, the third generation quarks have the bulk mass $|c|<\frac{1}{2}$. The other quarks have the bulk mass $\frac{1}{2}<|c|$. Therefore, the left-handed up-quark is localised toward the UV brane and the right-handed up-quark and top-quark are localised toward the IR brane.

In contrast, gauge bosons do not have a parameters like the bulk mass parameter, so the profile of the gauge boson mode functions are determined only by the metric. Massless gauge boson has exactly flat mode function and 1st KK gauge bosons are peaked toward the IR brane [27]. The same is true for other gauge bosons. Their behaviour are plotted in Figure 2.

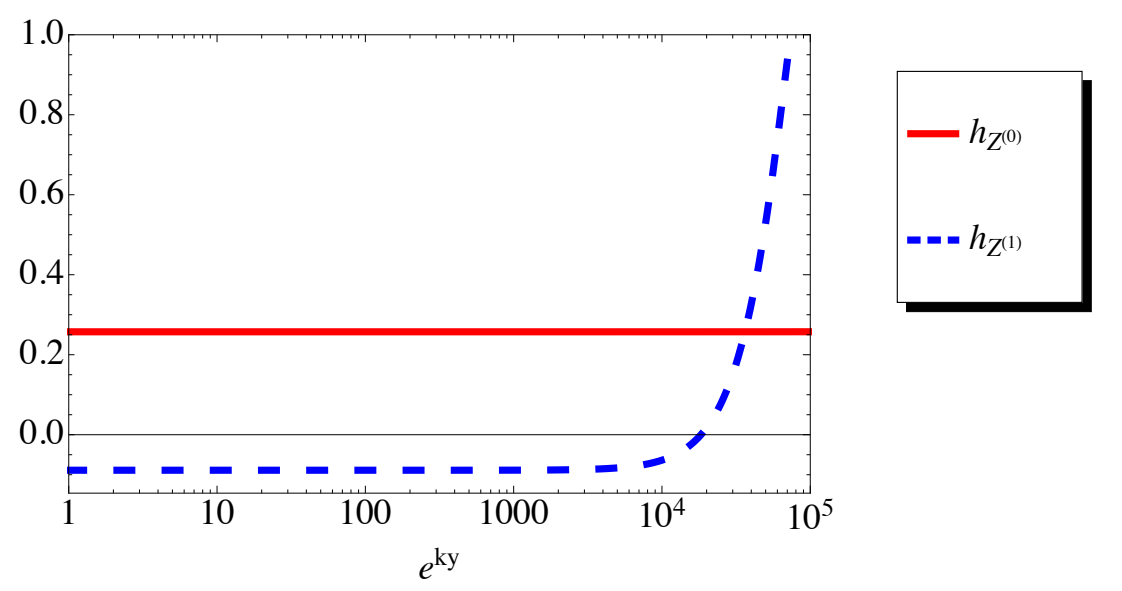

Figure 2: The profile of the $Z$-boson and 1st KK Z-boson mode functions are shown. The $Z$-boson is almost constant but very slightly peaked near the IR brane and the 1 st KK Z-boson is peaked near the IR brane.

$Z^{\prime}$ couplings are obtained by the overlap integral along the extra dimension. Because $Z^{\prime}$ mode functions are peaked towards the IR brane, $Z^{\prime}$ couplings with the fermions which is peaked towards the IR brane is large. Therefore $Z^{\prime}$ couplings depend on the sign of the bulk mass parameter. However the fermion masses does not depend on it. The product of the left- and right-handed fermion mode functions with the same bulk mass, $f_{L}^{(0)}(y) \times f_{R}^{(0)}(y)$ is invariant under changing the sign of the bulk mass parameter $c \rightarrow-c$. The mode functions of fermion KK modes are abbreviated but have same property. Thus, the Yukawa couplings and the masses are determined by the absolute values of the bulk mass parameters and independent of its sign. 
Table 3: Couplings of $Z$ and $Z^{(1)}$ bosons to electron and muon in unit of $g_{w} / \cos \theta_{W}=e /\left(\sin \theta_{W} \cos \theta_{W}\right)$ for $\theta_{H}=0.10$ and $\sin ^{2} \theta_{W}=0.23122$.

\begin{tabular}{|c|c|c|c|c|}
\hline$f$ & $g_{Z e}^{L}$ & $g_{Z e}^{R}$ & $g_{Z^{(1)} e}^{L}$ & $g_{Z^{(1)} e}^{R}$ \\
\hline SM & -0.26878 & +0.23122 & & \\
\hline$c_{e}>0$ & -0.26884 & +0.23133 & +0.09871 & +0.91478 \\
\hline$c_{e}<0$ & -0.26641 & +0.23377 & -1.05350 & -0.08584 \\
\hline$f$ & $g_{Z \mu}^{L}$ & $g_{Z \mu}^{R}$ & $g_{Z^{(1)}}^{L} \mu$ & $g_{Z^{(1)} \mu}^{R}$ \\
\hline SM & -0.26878 & +0.23122 & & \\
\hline$c_{\mu}>0$ & -0.26884 & +0.23133 & +0.09871 & +0.85881 \\
\hline$c_{\mu}<0$ & -0.26641 & +0.23377 & -0.98901 & -0.08584 \\
\hline
\end{tabular}

The electron and muon couplings with $Z$ and $Z^{(1)}$ bosons are shown in Table 3. As explained above, IR localised fermion couples with $Z^{\prime}$ largely. Therefore, for positive and negative $c_{l}$, the right- and left-handed lepton couplings are large, respectively. This large asymmetry of couplings cause large deviations of cross sections from that in the SM by using polarised beams as shown later. The deviations of the electron and muon couplings with $Z$ boson are $O(0.01) \%$ for $c_{l}>0$ and $O(0.1) \%$ for $c_{l}<0$. Consequently, the forward-backward asymmetry of $e^{+} e^{-} \rightarrow \mu^{+} \mu^{-}$at the $Z$-pole deviates nearly $3 \sigma$ for $c_{l}<0$ from the observed value. To obtain the forward-backward asymmetry consistent with the experiment, $\sin ^{2} \theta_{W} \simeq 0.2295$ is required. In the following, only $c_{l}>0$ is considered. The $Z^{(1)}$ boson mass and decay width are shown in Table 4 . For the complete set of the $Z^{\prime}$ mass, couplings and decay width, please see Ref. [13].

Table 4: Masses of $Z^{(1)}$ and total decay width of $\gamma^{(1)}$ in the unit of GeV. $\Gamma_{Z^{(1)}}( \pm, \pm)$ represent that left and right sign is sign of $c_{l}$ and $c_{q}$.

\begin{tabular}{c|ccccc}
$\theta_{H}$ & $m_{Z^{(1)}}$ & $\Gamma_{Z^{(1)}}(+,+)$ & $\Gamma_{Z^{(1)}}(+,-)$ & $\Gamma_{Z^{(1)}}(-,+)$ & $\Gamma_{Z^{(1)}}(-,-)$ \\
\hline 0.10 & 6585 & 429 & 1632 & 959 & 2162 \\
0.09 & 7149 & 463 & 1674 & 1014 & 2225 \\
0.08 & 7855 & 534 & 1705 & 1112 & 2283
\end{tabular}

\section{Cross section and forward-backward asymmetry}

In the following, $e^{+} e^{-} \rightarrow \bar{f} f(f \neq e)$ process is considered at the tree-level. Process mediated by Higgs boson is ignored, therefore the cross section of $e^{-} e^{+} \rightarrow Z^{\prime} \rightarrow \bar{f} f(f \neq e)$ is given by

$$
\frac{d \sigma}{d \cos \theta}=\frac{1}{4}\left[\left(1-P_{e^{-}}\right)\left(1+P_{e^{+}}\right) \frac{d \sigma_{L R}}{d \cos \theta}+\left(1+P_{e^{-}}\right)\left(1-P_{e^{+}}\right) \frac{d \sigma_{R L}}{d \cos \theta}\right]
$$

where $\sigma_{L R}\left(\sigma_{R L}\right)$ is $e_{L}^{-} e_{R}^{+}\left(e_{R}^{-} e_{L}^{+}\right) \rightarrow \bar{f} f$ cross section and $P_{e^{ \pm}}\left(-1 \leq P_{e^{ \pm}} \leq 1\right)$ is the longitudinal polarisation. The electron and positron is purely right-handed when $P_{e^{ \pm}}=1$. (4.1) is rewritten by $P_{\text {eff }}=\left(P_{e^{-}}-P_{e^{+}}\right) /\left(1-P_{e^{-}} P_{e^{+}}\right)$as $\sigma\left(P_{\text {eff }}, 0\right)=\sigma\left(P_{e^{-}}, P_{e^{+}}\right) /\left(1-P_{e^{-}} P_{e^{+}}\right)$, then the ratio of $\sigma$ is parametrised by one polarisation parameter $P_{\text {eff. Typical values of polarisation parameters are taken }}$ to $\left(P_{e^{-}}, P_{e^{+}}\right)=( \pm 0.8, \mp 0.3)$, where $P_{\text {eff }}= \pm 0.887$. 


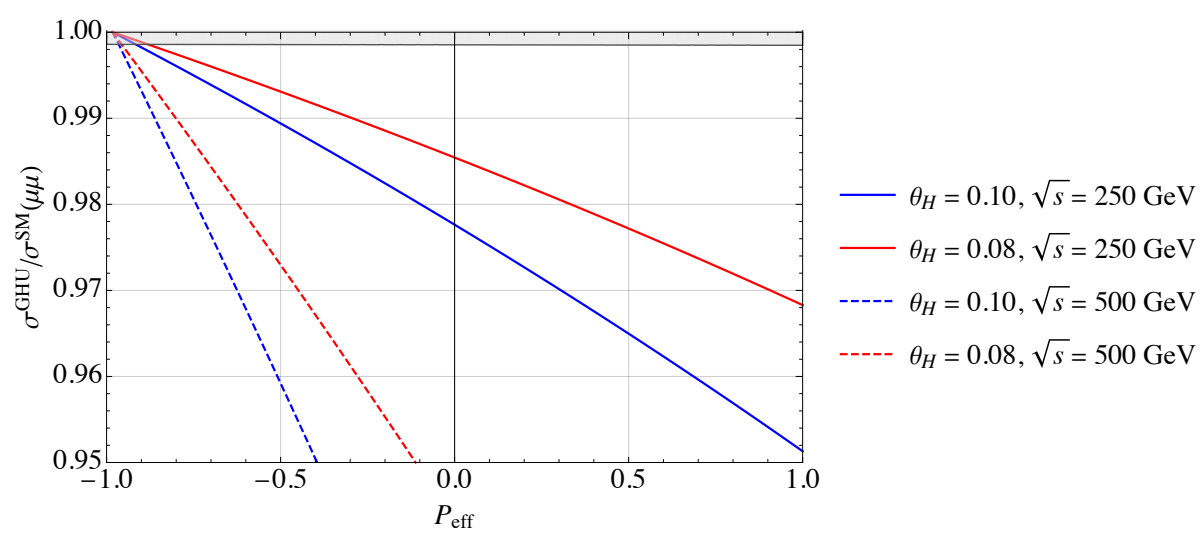

Figure 3: Ratio of the cross sections in the GHU model to that in the SM with polarisation beams for the $e^{+} e^{-} \rightarrow \mu^{+} \mu^{-}$process. Solid and dotted lines are for $\sqrt{s}=250 \mathrm{GeV}$ and $500 \mathrm{GeV}$, respectively. Blue-thick and red-thin lines correspond to $\theta_{H}=0.10$ and 0.08 , respectively. The grey band indicates the statistical uncertainty in the $\mathrm{SM}$ at $\sqrt{s}=250 \mathrm{GeV}$ with $250 \mathrm{fb}^{-1}$ data.

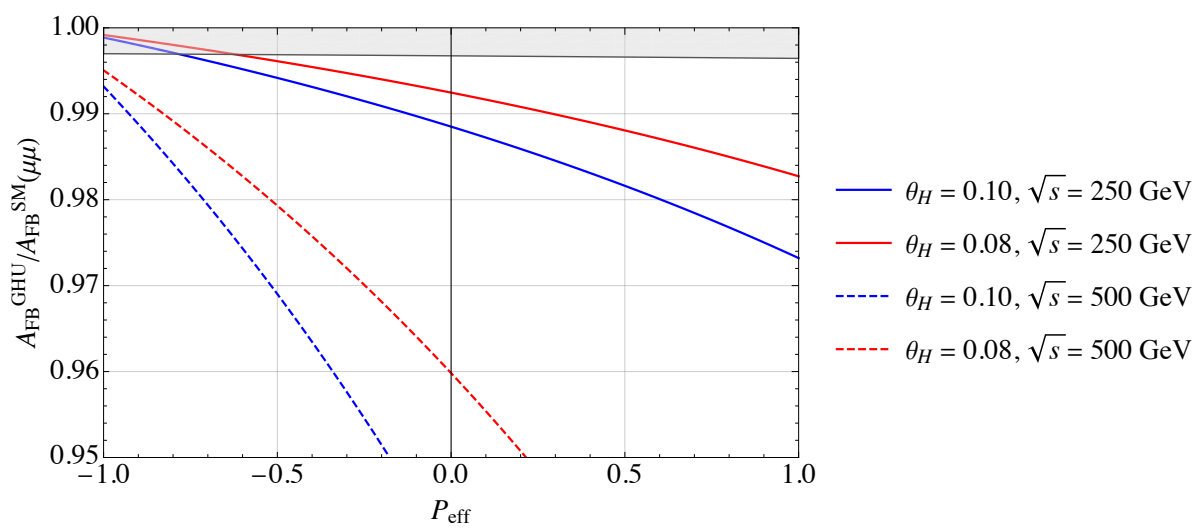

Figure 4: Ratio of the forward-backward asymmetry in the GHU model to that in the SM with polarisation beams for the $e^{+} e^{-} \rightarrow \mu^{+} \mu^{-}$process. Solid and dotted lines are for $\sqrt{s}=250 \mathrm{GeV}$ and $500 \mathrm{GeV}$, respectively. Blue-thick and red-thin lines correspond to $\theta_{H}=0.10$ and 0.08 , respectively. The grey band indicates the statistical uncertainty in the $\mathrm{SM}$ at $\sqrt{s}=250 \mathrm{GeV}$ with $250 \mathrm{fb}^{-1}$ data.

In the $e^{+} e^{-} \rightarrow \mu^{+} \mu^{-}$process, the difference between $c_{q}>0$ case and $c_{q}<0$ case is caused from only the $Z^{\prime}$ decay widths. Thus the difference of two cases is very small. The analysis of the $e^{+} e^{-} \rightarrow \mu^{+} \mu^{-}$process in the GHU model is shown in [12]. In the $\mathrm{SM}$ at $\sqrt{s}=250 \mathrm{GeV}$ with unpolarised (and $\left.\left(P_{e^{-}}, P_{e^{+}}\right)=(+0.8,-0.3)\right)$ beam, the cross section is $1.87 \mathrm{pb}(2.16 \mathrm{pb})$ and $4.66 \times 10^{5}\left(5.41 \times 10^{5}\right)$ events are expected with $250 \mathrm{fb}^{-1}$. By using unpolarised and polarised beam $\left(P_{\text {eff }}=+0.887\right)$, the deviations of the cross section in the GHU from the SM are about $-2.2 \%$ and $-4.6 \%$ as shown in Fig. 3. The forward-backward asymmetry is shown in Fig. 4. $0.32 \%$ statistical error is expected in the $\mathrm{SM}$ at $\sqrt{s}=250 \mathrm{GeV}$ with $250 \mathrm{fb}^{-1}$ beam. At $\theta_{H}=0.10$, $\sqrt{s}=250 \mathrm{GeV}$ with $250 \mathrm{fb}^{-1}$, the deviation is nearly 4 times larger than the statistical error by unpolarised beam and the deviation is nearly 8 times larger than the statistical error by polarised beam $P_{\text {eff }}=+0.887$. For $\theta_{H}=0.08$, the deviation is $-1.6 \%$, nearly 5 times larger than the statistical error by polarised beam. 
For the forward-backward asymmetry of $e^{+} e^{-} \rightarrow \bar{c} c$ process, its statistical error of the SM predictions are about $0.16 \%$ at $250 \mathrm{GeV}$ with unpolarised $250 \mathrm{fb}^{-1}$ luminosity beam. The ratio of that in the GHU and that in SM plotted in Fig. 5. Blue-thick and red-thin lines correspond to the positive bulk mass and $\theta_{H}=0.10$ and 0.08 case, black-thick and green-thin lines correspond to the negative bulk mass and $\theta_{H}=0.10$ and 0.08 case, respectively. For the positive bulk mass case, $A_{\mathrm{FB}}^{\mathrm{GHU}}(\bar{c} c)$ is larger than that in the SM. For $\theta_{H}=0.10$ and $\sqrt{s}=250 \mathrm{GeV}$, the deviation is nearly 2 times larger than the statistical error for $P_{\text {eff }} \geq 0$. For negative bulk mass case, the deviation 10 times larger than the statistical error is predicted at $\theta_{H}=0.10$ and $\sqrt{s}=250 \mathrm{GeV}$ with $P_{\text {eff }}=+0.887$. At $\theta_{H}=0.08$ and $\sqrt{s}=250 \mathrm{GeV}$ with $P_{\text {eff }}=+0.887$, the deviation is 6.2 times larger than the statistical error.

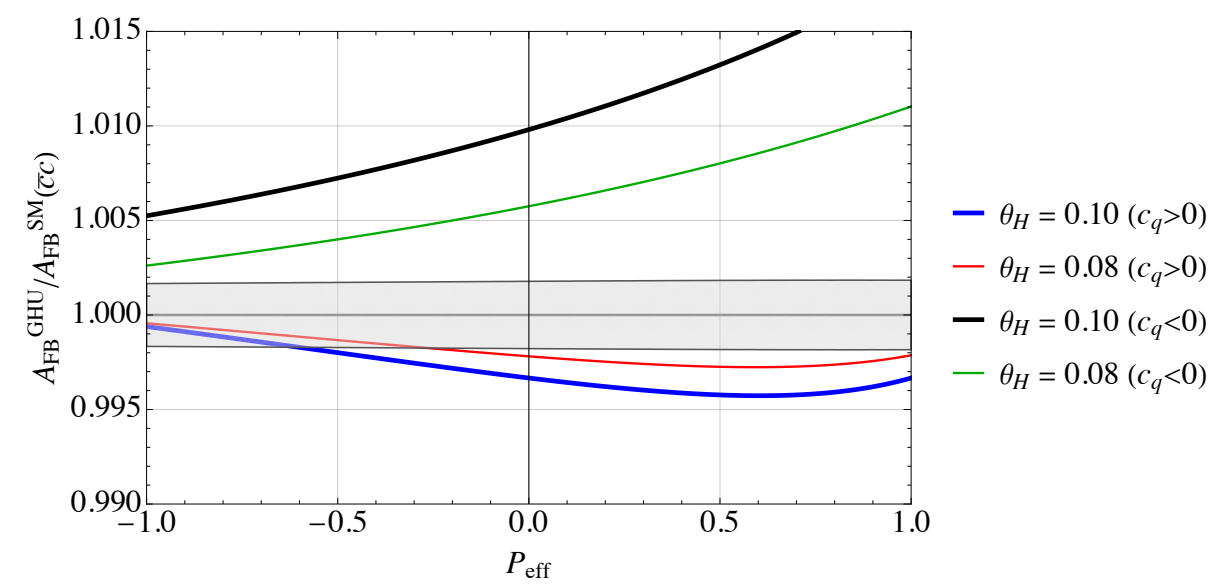

Figure 5: Ratio of the forward-backward asymmetry in the GHU model to that in the SM with polarisation beams for the $e^{+} e^{-} \rightarrow \bar{c} c$ process. Blue-thick and red-thin lines correspond to the positive bulk mass and $\theta_{H}=0.10$ and 0.08 case, black-thick and green-thin lines correspond to the negative bulk mass and $\theta_{H}=$ 0.10 and 0.08 case, respectively. The gray band indicates the statistical uncertainty in the SM at $\sqrt{s}=$ $250 \mathrm{GeV}$ with $250 \mathrm{fb}^{-1}$ data.

For the $e^{+} e^{-} \rightarrow \bar{b} b$ process, both the left- and right-handed bottom-quark couples to $Z^{\prime}$ largely, regardless of the sign of the bulk mass. The forward-backward asymmetry of $e^{+} e^{-} \rightarrow \bar{b} b$ process at $\sqrt{s}=250 \mathrm{GeV}$ in the GHU increase from that in the SM both in $c_{q}>0$ and $c_{q}<0$ cases. In the GHU model $A_{\mathrm{FB}}^{\mathrm{GHU}, c_{q}>0}(\bar{b} b)$ increase $4.24 \%$ and $4.15 \% A_{\mathrm{FB}}^{\mathrm{GH}, c_{q}<0}(\bar{b} b)$ increase $7.33 \%$ and $3.83 \%$ from that in the SM for $\theta_{H}=0.10$ and 0.08 cases at $\sqrt{s}=250 \mathrm{GeV}$ with $P_{\text {eff }}=+0.887$, respectively. At $\sqrt{s}=250 \mathrm{GeV}$ with $P_{\text {eff }}=+0.887$ and $250 \mathrm{fb}^{-1}$ data, the statistical error of $A_{\mathrm{FB}}^{\mathrm{SM}}(\bar{b} b)$ is $0.7 \%$. Therefore the deviations of $A_{\mathrm{FB}}^{\mathrm{GHU}}(\bar{b} b)$ are more than 5 times larger than the statistical error.

The differential cross section in the GHU model is studied currently. At $\sqrt{s}=250 \mathrm{GeV}$ with $P_{\text {eff }}=+0.887$ and $250 \mathrm{fb}^{-1}, 5.8 \times 10^{4}$ events are predicted in the SM for the bin $\cos \theta=$ $[+0.8,+0.9]$, which leads to the statistical error $0.41 \%$. At the same condition, the GHU predicts the deviation 13.3 and 8.6 times the statistical error for $\theta_{H}=0.10$ and 0.08 , respectively. The ratio of the differential cross section in the GHU model to that in the SM with $P_{\text {eff }}=+0.887$ is plotted in Fig .6. 


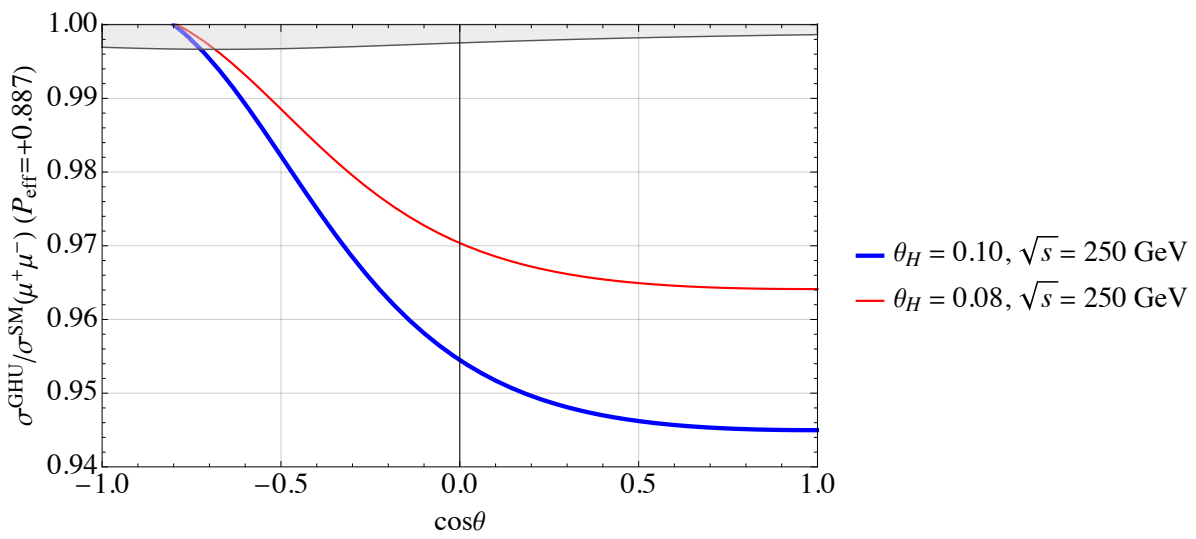

Figure 6: Ratio of the differential cross section in the GHU model to that in the SM with $P_{\text {eff }}=+0.887$ for the $e^{+} e^{-} \rightarrow \mu^{+} \mu^{-}$process. Blue-thick and red-thin lines correspond to the positive bulk mass and $\theta_{H}=0.10$ and 0.08 case, respectively. The gray band indicates the statistical uncertainty in the $\mathrm{SM}$ at $\sqrt{s}=250 \mathrm{GeV}$ with $250 \mathrm{fb}^{-1}$ data.

\section{Summary}

In this proceedings, $Z^{\prime}$ effects on the cross sections and forward-backward asymmetries in the $S O(5) \times U(1)$ GHU A-model are explained. Because of the behaviours of the mode functions, left(right-)handed fermions with positive (negative) bulk mass term couple with $Z^{\prime}$ largely. Therefore by using the polarised beam, the significant deviations of the cross sections and forward-backward asymmetries from those in the $\mathrm{SM}$ are predicted at $\sqrt{s}=250 \mathrm{GeV}$ although the $Z^{\prime}$ mass is $6-8 \mathrm{TeV}$. Other observables such as the left-right asymmetry and differential cross section is to be studied. In this analysis, the lepton bulk mass parameters are set to be positive but it can be negative. Therefore $Z^{\prime}$ effects should be more deeply clarified in both the $S O(5) \times U(1)$ GHU A-model and B-model. I am going to study on these topics in near future.

\section{Acknowledgements}

This work is supported by the National Natural Science Foundation of China (Grant Nos. 11775092, 11675061, 11521064, 11435003 and 11947213), and the International Postdoctoral Exchange Fellowship Program (IPEFP).

\section{References}

[1] Y. Hosotani, "Dynamical Mass Generation by Compact Extra Dimensions", Phys. Lett. 126B, 309 (1983).

Y. Hosotani, "Dynamics of Nonintegrable Phases and Gauge Symmetry Breaking", Ann. Phys. 190, 233 (1989).

[2] A. T. Davies and A. McLachlan, "Gauge Group Breaking By Wilson Loops", Phys. Lett. B 200 (1988) 305.

A. T. Davies and A. McLachlan, "Congruency Class Effects in the Hosotani Model", Nucl. Phys. B 317 (1989) 237. 
[3] H. Hatanaka, T. Inami and C. S. Lim, "The Gauge hierarchy problem and higher dimensional gauge theories", Mod. Phys. Lett. A 13, 2601 (1998).

H. Hatanaka, "Matter representations and gauge symmetry breaking via compactified space", Prog. Theor. Phys. 102, 407 (1999).

[4] K. Agashe, R. Contino and A. Pomarol, “The Minimal composite Higgs model”, Nucl. Phys. B 719, 165 (2005).

[5] R. Contino, L. Da Rold and A. Pomarol, "Light custodians in natural composite Higgs models", Phys. Rev. D 75, 055014 (2007).

[6] A. D. Medina, N. R. Shah and C. E. M. Wagner, "Gauge-Higgs Unification and Radiative Electroweak Symmetry Breaking in Warped Extra Dimensions”, Phys. Rev. D 76 (2007) 095010.

[7] Y. Sakamura and Y. Hosotani, " $W W Z, W W H$, and $Z Z H$ Couplings in the Dynamical Gauge-Higgs Unification in the Warped Spacetime", Phys. Lett. B 645, 442 (2007).

[8] Y. Hosotani, K. Oda, T. Ohnuma and Y. Sakamura, "Dynamical Electroweak Symmetry Breaking in $S O(5) \times U(1)$ Gauge-Higgs Unification with Top and Bottom Quarks”, Phys. Rev. D 78, 096002 (2008), Erratum: [Phys. Rev. D 79, 079902 (2009)].

[9] S. Funatsu, H. Hatanaka, Y. Hosotani, Y. Orikasa and T. Shimotani, "Novel universality and Higgs decay $H \rightarrow \gamma \gamma, g g$ in the $S O(5) \times U(1)$ gauge-Higgs unification”, Phys. Lett. B 722, 94 (2013).

[10] S. Funatsu, H. Hatanaka and Y. Hosotani, " $H \rightarrow Z \gamma$ in the gauge-Higgs unification", Phys. Rev. D 92, no. 11, 115003 (2015), Erratum: [Phys. Rev. D 94, no. 1, 019902 (2016)].

[11] S. Funatsu, H. Hatanaka, Y. Hosotani and Y. Orikasa, "Collider signals of $W^{\prime}$ and $Z^{\prime}$ bosons in the gauge-Higgs unification”, Phys. Rev. D 95, no. 3, 035032 (2017).

[12] S. Funatsu, H. Hatanaka, Y. Hosotani and Y. Orikasa, "Distinct signals of the gauge-Higgs unification in $e^{+} e^{-}$collider experiments", Phys. Lett. B 775, 297 (2017).

[13] S. Funatsu, "Forward-backward asymmetry in the gauge-Higgs unification at the International Linear Collider", Eur. Phys. J. C 79, no. 10, 854 (2019).

[14] S. Funatsu, H. Hatanaka, Y. Hosotani, Y. Orikasa and N. Yamatsu, "GUT inspired $S O(5) \times U(1) \times S U(3)$ gauge-Higgs unification”, Phys. Rev. D 99 (2019) no.9, 095010.

[15] J. Yoon and M. E. Peskin, "Competing forces in five-dimensional fermion condensation", Phys. Rev. D 96, no. 11, 115030 (2017).

[16] J. Yoon and M. E. Peskin, "Dissection of an $S O(5) \times U(1)$ gauge-Higgs unification model”, Phys. Rev. D 100, no. 1, 015001 (2019).

[17] H. Baer et al., "The International Linear Collider Technical Design Report - Volume 2: Physics", arXiv:1306.6352 [hep-ph].

[18] D. M. Asner et al., "ILC Higgs White Paper", arXiv:1310.0763 [hep-ph].

[19] H. Aihara et al., "The International Linear Collider. A Global Project”, arXiv:1901.09829 [hep-ex].

[20] P. Bambade et al., "The International Linear Collider: A Global Project”, arXiv:1903.01629 [hep-ex].

[21] M. S. Amjad et al., "A precise characterisation of the top quark electro-weak vertices at the ILC", Eur. Phys. J. C 75, no. 10, 512 (2015).

[22] S. Bilokin, R. Pöschl and F. Richard, "Measurement of b quark EW couplings at ILC", arXiv: 1709.04289 [hep-ex]. 
[23] W. Bernreuther, L. Chen, I. García, M. Perelló, R. Pöschl, F. Richard, E. Ros and M. Vos, "CP-violating top quark couplings at future linear $e^{+} e^{-}$colliders", Eur. Phys. J. C 78, no. 2, 155 (2018).

[24] F. Richard, "Bhabha scattering at ILC250", arXiv:1804.02846 [hep-ex].

[25] L. Randall and R. Sundrum, “A Large mass hierarchy from a small extra dimension”, Phys. Rev. Lett. 83, 3370 (1999).

[26] Y. Grossman and M. Neubert, "Neutrino masses and mixings in nonfactorizable geometry", Phys. Lett. B 474, 361 (2000).

[27] A. Pomarol, "Gauge bosons in a five-dimensional theory with localized gravity", Phys. Lett. B 486, 153 (2000). 